\title{
Evaluating Performance of Multiplex Real Time PCR for the Diagnosis of Malaria at Elimination Targeted Low Transmission Settings of Ethiopia.
}

\section{Mahlet Belachew}

Addis Ababa University College of Health Sciences

\section{Mistire Wolde}

Addis Ababa University College of Health Sciences

\section{Desalegn Nega}

Ethiopian Public Health Institute

\section{Bokretsion Gidey}

Ethiopian Public Health Institute

\section{Legessie Negash}

Ethiopian Public Health Institute

\section{Ashenafi Assefa}

Ethiopian Public Health Institute

\section{Geremew Tasew}

Ethiopian Public Health Institute

\section{Adugna Woyessa}

Ethiopian Public Health Institute

Adugna Abera Hirpa ( $\nabla$ adugna.abera2020@gmail.com )

Ethiopian Public Health Institute https://orcid.org/0000-0001-6572-8113

\section{Research}

Keywords: Malaria elimination, Multiplex real time PCR, Diagnostic performance

Posted Date: June 2nd, 2021

DOl: https://doi.org/10.21203/rs.3.rs-572301/v1

License: (c) (i) This work is licensed under a Creative Commons Attribution 4.0 International License. Read Full License

Version of Record: A version of this preprint was published at Malaria Journal on January 6th, 2022. See the published version at https://doi.org/10.1186/s12936-021-04029-x. 


\section{Abstract}

Background: Malaria incidence has declined in Ethiopia in the past ten years. Current malaria diagnostic tests, including light microscopy and antigen-detecting rapid tests (RDTs) cannot reliably detect lowdensity infections. Studies have shown that nucleic acid amplification tests are highly sensitive and specific in detecting malaria infection. Thus, this study took place with the aim of evaluating the performance of multiplex real time PCR for the diagnosis of malaria using patient samples collected from health facilities located at malaria elimination targeted low transmission settings in Ethiopia.

Methods: A health facility based cross sectional survey was conducted in selected malaria sentinel sites. Malaria suspected febrile outpatients referred to laboratory for malaria testing between December 2019 and March 2020 were enrolled into this study. Socio demographic information and capillary blood samples were collected from the study participants and tested at spot with RDTs. Additionally, five circles of dry blood sample (DBS) samples on Whatman filter paper and thick and thin smear were prepared for molecular testing and microscopic examination, respectively. Multiplex real time PCR assay was performed at EPHI malaria laboratory. The performance of multiplex real time PCR assay, microscopy and RDT for the diagnosis of malaria was compared and evaluated against each other.

Results: Out of 271 blood samples, multiplex real time PCR identified 69 malaria cases as $P$. falciparum infection, 16 as $P$. vivax and 3 as mixed infections. Of the total samples, light microscopy detected 33 as Pf, 18 as PV and RDT detected 43 as Pf, 17 as PV, and one mixed infection. Using light microscopy as reference test, the sensitivity and specificity of multiplex real time PCR were 100\% (95\% Cl [93-100]) and 83.2\% (95\% $\mathrm{Cl}$ [77.6-87.9]), respectively. Using multiplex real time PCR as a reference, light microscopy and RDT had sensitivity of 58\% (95\% Cl [46.9-68.4] and 67\% (95\% Cl [56.2-76.7]); and 100\% (95\% Cl [98$100]$ and 98.9 (95\% $\mathrm{Cl}$ (96-99.9), respectively. Substantial level of agreement was reported between microscopy and multiplex real time PCR results with kappa value of 0.65 .

Conclusions: Multiplex real time PCR had an advanced performance in parasite detection and species identification on febrile patients' samples than did microscopy and RDT in low malaria transmission settings. It is highly sensitive malaria diagnostic method that can be used in malaria elimination program, particularly for community based epidemiological samples. Although microscopy and RDT had reduced performance when compared to multiplex real time PCR, still had an acceptable performance in diagnosis of malaria cases on patient samples at clinical facilities.

\section{Background}

Malaria is a life-threatening disease caused by the genus Plasmodium. Currently, there are five species of the genus Plasmodium known to cause human malaria: Plasmodium falciparum (P. falciparum), $P$. vivax, $P$. ovale, $P$. malariae and $P$. knowlesi. Malaria parasite is transmitted by the bite of infected female Anopheles mosquitoes. In Plasmodium lifecycle, the sporozoite stages are injected into the human host by mosquito bite, migrate via blood stream to liver and infect the hepatocytes. In the hepatocytes, the 
sporozoites develop into mature schizonts and release merozoites to blood, with some sporozoites remain dormant (called hypnozoites) inside the liver cells in the case of vivax and ovale infections for future malaria periodic relapse. The merozoites infect erythrocytes while certain portion of the merozoites develop into sexual forms (gametocytes) which are ingested by a blood feeding mosquito, thereby completing the lifecycle. Plasmodium infection causes an acute febrile illness after 7 days or longer period of incubation. The infected individual more commonly presents with clinical symptom of fever, chills, and headache. If malaria infection left untreated, it may possibly progress to severe and complicated cases which may lead to death of the patient $(1,2)$.

Within the last decade, the incidence and malaria specific mortality rate has declined worldwide due to concerted global malaria control efforts. According to the WHO malaria report 2018, the number of malaria cases in 2017 was 43 million fewer than the cases in 2000 and 20 million fewer than the cases in 2010. Malaria burden reduced substantially in the WHO African Region since 2010 mainly due to the scaled-up of malaria control interventions, such as long-lasting insecticide-treated mosquito nets (LLINs), indoor residual spraying (IRS), and treatment of $P$. falciparum infections with artemisinin-based combination therapy and accurate diagnosis of suspected cases $(3,4)$.

Malaria is significant public health challenge in Ethiopia since long time ago. However, in the last decade, the morbidity and mortality due to malaria infection has declined significantly. Though the first malaria eradication platform in Ethiopia was implemented in 1959 following the major malaria epidemic that hit the country in 1958, which caused 150,000 deaths, a significant decline in malaria morbidity and mortality in Ethiopia observed since 2005 after the implementation of scaling-up malaria prevention and control interventions in 2004. The last epidemic was occurred in 2003 that caused 3,000 deaths and 2 million clinical malaria cases. Between 2004 and 2007, malaria cases and deaths in all age groups declined by 55\%. A survey conducted in 2006/2007 in three regions: Amhara, Oromia, and Southern Nations Nationalities and Peoples' Region (SNNPR) showed malaria prevalence of $4.1 \%$. According to 2007 and 2011 Ethiopian malaria indicator survey (EMIS), malaria prevalence by microscopy was around $1 \%$ in all age groups in rural malarious areas during the peak transmission season $(5,6)$.

According to Federal ministry of health $(\mathrm{FMoH})$, confirmed malaria cases in 2018 were 989,182 which is significantly lower than the cases reported in 2014 which were 2,210,298 (7). Based on this remarkable decline in malaria incidence and morbidity, the 2021-2025 national malaria strategic plan (NMSP) set a goal to achieve zero indigenous malaria in selected low transmission areas by 2025 and national elimination by 2030 (8).

Malaria transmission in Ethiopia is irregular; in most parts of the country, it is highly seasonal; but in some other parts of the country, nearly constant transmission is observed. The transmission peaks biannually, the major malaria transmission occur from September to December, following rainy season from June to August, while the minor transmission season occurs between April and May following the February to March rains. In general, malaria in Ethiopia has low and unstable transmission patterns that result in low herd immunity and significant clinical malaria illness risk after malaria infections, increased 
tendency for rapid progression to severe malaria, and propensity for malaria epidemics affecting all age groups of the community. P. falciparum and $P$. vivax are the two most dominant malaria parasites in Ethiopia which are transmitted by the bite of Anopheline mosquitos. A. arabiensis are the main vectors whereas $A$. pharoensis, $A$. funestus and $A$. nili are also widely distributed species in the country for malaria transmission (9).

In the progress towards malaria elimination, the transmission has declined and the detection of infections become challenging in low transmission settings because of low parasite load (10). In order to achieve malaria elimination, it is critical to detect all infections, including those with low and submicroscopic carriers as they constitute reservoirs in the community for significant proportion of mosquito infection and are responsible for an ongoing transmission. Therefore, malaria elimination programs will have been needing highly sensitive and robust diagnostic tools for mass screening and surveillance purposes (11).

Light microscopy using Giemsa-stained blood films and RDTs are primary malaria diagnostic tools at health facilities. Light microscopy is considered as gold standard for malaria parasite identification and confirmation at hospitals and health centers all over Ethiopia. In malaria endemic peripheral and rural areas, RDTs used as malaria diagnostic tools centered at health posts (12). RDTs are immunechromatographic tests, identifying Plasmodium specific histidine reach protein-2 (HRP-2), lactate dehydrogenase protein (LDH) and aldolase. RDTs have improved sensitivity and specificity for detection of Plasmodium infections, require no electric source, no need of expertise to read the result and reduce time that required for performing a test, compared to microscopy. Bias during reading the result band and false negative result during hyperparasitaemia due to antigen prozone effect are limitations of RDT tests. Moreover, during mixed and low parasitaemia infection cases, both microscopy and RDTs have shown reduced performance for detection of Plasmodium (13). Now a day, different molecular tests are in use for the detection of Plasmodium species.

These molecular tests have been developed mostly based on real time quantitative PCR, with qualities of automated, quantitative and closed system that reduces the risk of contamination inherent in conventional PCR. Multiplex real time PCR has improved capability for the detection of Plasmodium species in low parasitaemia cases; usually it has the detection limit of less than 2 parasites per microliter and has an advantage of simultaneous detection of mixed Plasmodium infections, compared to microscopy and RDTs $(10,14)$.

\section{Materials And Methods}

\section{Study area}

The study was conducted in two malaria sentinel sites in Ethiopia namely Shewa Robit health centre at North East and Metehara health center at Eastern Ethiopia. The health care facilities are located in the malaria elimination targeted areas of Ethiopia. EPHI in collaboration with Federal Ministry of Health 
established 25 malaria sentinel surveillance sites, representing regional malaria cover in Ethiopia. These sites are eco-epidemiologically representative and focal area of malaria infection.

\section{Study design and period}

Facility based cross sectional study was conducted from December, 2019 to March, 2020 on malaria suspected febrile outpatients referred to the laboratory.

\section{Participant Selection Criteria}

\section{Inclusion criteria}

All malaria suspected febrile patients of any age referred to the laboratory for malaria testing were included in this study.

\section{Exclusion criteria}

Patients who take antimalarial therapy in the past 4 weeks before sample collection and critically ill patients were excluded from the study.

\section{Sample Size Calculation And Sampling Technique}

Sample size calculation

Sample size was calculated using Buderer's formula (15) as follows:

$Z^{2}{ }_{1-a / 2} \times S_{N} \times\left(1-S_{N}\right)$

$L^{2} \times$ Prevalence

$Z_{1-a / 2}$ (standard normal deviate corresponding to the specified size of the critical region $(a)=1.96, S N$ (anticipated sensitivity) $=0.9$, Prevalence $=13 \%, \mathrm{~L}$ (absolute precision desired on either side of sensitivity) $=0.1$. Because we couldn't find previous malaria prevalence studies done using multiplex real time PCR, we estimated (intellectual guess) malaria prevalence of $13 \%$ and the anticipated sensitivity of this method as $90 \%(95 \% \mathrm{Cl}: 80-100 \%)$ for $P$. falciparum, compared to conventional PCR. Then, a total of 271 participants were enrolled into this study.

\section{Sampling technique}

Convenient sampling technique was used on consecutive basis to recruit the study subjects referred to the laboratory for malaria testing. 


\section{Data Collection Procedure}

After getting patient consents, demographic profiles and clinical data were collected using structured questionnaire. Using capillary blood taken from each patient, thick and thin smears were prepared for microscopy. RDT testing was performed at spot and five circles of dried blood spot (DBS) were collected on Whatman filter paper and transported by cold chain to EPHI laboratory for the molecular tests.

\section{Laboratory Analysis}

Rapid diagnostic test (RDT)

RDT testing was performed as per the manufacturer instruction using CareStart ${ }^{\text {TM }}$ malaria HRP2/pLDH combo test. This test detects HRP2 and pLDH proteins specific to falciparum and vivax, respectively. The tests were performed in the field laboratory by health centers' laboratory personnel as a routine malaria testing.

\section{Malaria microscopy}

After preparation of thick and thin blood films, slides were allowed to air-dry at room temperature and fixed using absolute methanol then stored at $2-8^{\circ} \mathrm{C}$ until transported to EPHI. In the EPHI parasitology laboratory, slides were stained with $10 \%$ Giemsa solution for 10 minutes, after being air dried, both thick and thin smear were examined by an experienced laboratory technologist. An expert microscopist rechecked all positive slides, and $10 \%$ of negative slides. According to WHO malaria microscopy standard operating procedure, at least 100 high power fields (HPFs) were examined for parasites detection.

\section{DNA extraction}

Genomic DNA extraction was performed using QiagenQIAamp ${ }^{\circledR} 96$ DNA Blood Kit (QIAGEN Inc.) from DBS sample. Briefly, three 3-mm circles of the DBS punched out and placed into a 1.5-mL tube for processing as per manufacturer instructions. Finally, with $100 \mu$ l volume of elution buffer the DNA was eluted and stored at $-20^{\circ} \mathrm{C}$ until assayed.

\section{Multiplex real time PCR}

The PCR amplification was done by using primer and probes that targeting Plasmodium genus specific small unit of ribosomal RNA (18S rRNA), specific small unit of ribosomal RNA (P.v18S rRNA), and $P$. falciparum specific var gene acidic terminal sequence (varATS) (16). TaqMan fluorescence based DNA amplification and detection were performed using QuantStudio 5 Real time PCR system. For this study, the multiplex real time PCR assay were run in two round during the first run all samples were tested by multiplexing Pan-plasmodium specific and falciparum specific primers and the second were by multiplexing $P$. falciparum and $P$. vivax specific primers. Briefly, each reaction mixture was prepared by mixing $2 \mu \mathrm{l}$ of purified DNA template, $5 \mu$ l Luna Universal Probe qPCR Master Mix (New England Biolabs, 
Inc.), $2 \mu$ I PlasQ Primer Mix and $1 \mu \mathrm{l}$ molecular biology grade $\mathrm{H}_{2} \mathrm{O}$ with a final reaction mixture volume of $10 \mu \mathrm{l}$. Amplifications were carried out using thermal cycling conditions: for the first PCR run $95^{\circ} \mathrm{C}$ for 1 minute, followed by 45 cycles of $95^{\circ} \mathrm{C}$ for 15 seconds and $57^{\circ} \mathrm{C}$ for 45 seconds and for the second run $95^{\circ} \mathrm{C}$ for 1 minute, followed by 45 cycles of $95^{\circ} \mathrm{C}$ for 15 seconds and $53^{\circ} \mathrm{C}$ for 45 seconds. The 3D7 DNA standard was run in each experiment and used as a positive control and nuclease free water used for a negative control.

Table 1

Primers and probes sequences used for qPCR assays in this study.

\begin{tabular}{|llll|}
\hline Target Gene & Oligo sequence & Fluorophores & TM •C \\
\hline Pspp18S F & GCTCTTTCTTGATTTCTTGGATG & & 51.71 \\
\hline Pspp18S R & AGCAGGTTAAGATCTCGTTCG & & 52.4 \\
\hline Pspp18S Cy5 & ATGGCCGTTTTTAGTTCGTG & Cyanine 5 & 52 \\
\hline PfvarATS F & CCCATACACAACCAAYTGGA & & 51.78 \\
\hline PfvarATS R & TTCGCACATATCTCTATGTCTATCT & & 52.76 \\
\hline PfvarATS FAM & TRTTCCATAAAGGT 5'-3' & Fluorescein & NA \\
\hline Pv18S F & ACTAGGCTTTGGATGAAAGATTTTA & & 53.23 \\
\hline Pspp18S R & AACCCAAAGACTTTGATTTCTCATAA & & 51.65 \\
\hline Pv18S probe & GAATTTTCTCTTCGGAGTTTAT & Cy5-BHQ2 & 46 \\
\hline HsRNaseP F & AGATTTGGACCTGCGAGCG & & 53.25 \\
\hline HsRNaseP R & GAGCGGCTGTCTCCACAAGT & & 55.88 \\
\hline HsRNaseP_1 & TTCTGACCTGAAGGCTCTGCGCG & HEX & 60.62 \\
\hline
\end{tabular}

\section{Data Quality Assurance}

Onsite training was given to all data collectors. All blood films, DBS samples and RDT testing were performed based on standard operating procedures. The quality of each reagent was cheeked before the laboratory analysis was performed. Samples and reagents were stored at appropriate temperature as indicted on the manufacturer inserts. Internal and external quality controls were run as required during analysis, all remaining samples stored at $-20^{\circ} \mathrm{C}$, the collected data were checked for its consistency and completeness before any attempt to enter code and analyze the data. Data was double checked manually for completeness and consistency before data entry. Finally, Epi-Info version-7 was used to control and manage errors resulting from data entry process.

\section{Data Analysis And Interpretation}


The collected data were coded, entered into Epi-Info version-7, and was exported to STATA version 20 software before analysis and interpretation. Descriptive statistics was used to describe patient's socio demographic and clinical characteristics. The sensitivity, specificity, predictive values and kappa coefficient was estimated by comparing results from all three assays and $95 \%$ confidence interval was computed.

\section{Ethical Considerations}

This study was approved by Institutional Ethical Review board of College of Health Sciences, Addis Ababa University and Scientific and ethical review office of EPHI (Protocol number: EPHI-IRB-219-2019). Official letter was written to sentinel sites. The confidentiality of patient related data was maintained by avoiding possible identifiers such as name of the patient. Finally, after the whole process of data collection, all data was kept safe throughout the whole process of the research work.

\section{Results}

\section{Socio demographic characteristics of study participants}

A total of 271 study participants were enrolled in this study. The mean age of the patients was 24.12 years ( $\pm 14.83 \mathrm{SD}$ ) with 4 months minimum age and 90 years maximum age. More than half of the participants were females (54.6\%) (Table 2).

Table 2

Age and sex distribution of the study participants

\begin{tabular}{|llll|}
\hline Age group & Female, $\mathbf{N}(\%)$ & Male, $\mathbf{N}(\%)$ & Total \\
\hline $0-5$ & $14(5.2)$ & $11(4.1 \%)$ & 25 \\
\hline $6-15$ & $23(8.5)$ & $20(7.4 \%)$ & 43 \\
\hline $16-25$ & $72(26.6)$ & $34(12.5 \%)$ & 106 \\
\hline $26-40$ & $28(10.3)$ & $44(16.2 \%)$ & 72 \\
\hline $41-64$ & $6(2.2)$ & $13(4.8 \%)$ & 19 \\
\hline$\geq 65$ & $5(1.8)$ & $1(0.4)$ & 6 \\
\hline Total & $148(54.6 \%)$ & $123(45.4 \%)$ & 271 \\
\hline
\end{tabular}

\section{Clinical Characteristics Of Study Participants}

On presentation, 266(98.15\%) of the study participants reported they had fever, $253(93.36 \%)$ had chills, and 253(93.36\%) had headache. Besides, sweating and muscle pain was diagnosed in 207 (76.38\%) and 
199(73.43\%) of the cases, respectively. None of the patients had shown severe malaria sign and symptoms during the study period (data not shown).

\section{Laboratory results of study participants by multiplex real time PCR, microscopy and RDT}

Among 271 study participants, malaria positive cases by microscopy, RDT and multiplex real time PCR methods were respectively 51 (19\%), 66 (22.5\%) and 88 (32.5\%) of Plasmodium species. The positivity rate by three of the methods was increased among the age group of 16-25 years, and in male participants (Table 3). From the total 271 study patients, $26.2 \%$ (71) were enrolled from Shewa Robit and the remaining $73.8 \%$ (200) from Metahara health centre. Besides, multiplex real time PCR detected $36.6 \%$ (26/71) of malaria positive patients in Shewa Robit and 31\% (62/200) positives in Metahara. Higher number of $P$. vivax (14 cases) was reported from Metahara health centre than 2 P.vivax cases in Shewa Robit. Comparing the three methods, the positivity rate was highest for multiplex real time-PCR that was $32.5 \%$ (88/271), followed by RDT $22.5 \%$ (61/271), and microscopy $18.8 \%(51 / 271)$, respectively. Three mixed infections ( $P$. falciparum and $P$. vivax) were detected by multiplex real time-PCR, one by RDT and none by microscopic method. There was little difference among the three methods in detecting $P$. vivax (RDT: 17, microscopy: 18 and multiplex PCR: 16). However, significant numbers of $P$. falciparum cases were positive by multiplex real time PCR than RDT and microscopy (69 vs 43 and 69 vs 33).

Among 271 participants, 266 patients were clinically presented with fever; out of these 50 were tested positive by microscopy, 60 by RDT and 87 by multiplex real time PCR for Plasmodium species (Table 3). 
Table 3

Malaria parasite positivity by RDTs, microscopy and multiplex real time PCR techniques among the sociodemographic and clinical variables, Dec to March 2020, Ethiopia.

\begin{tabular}{|c|c|c|c|c|}
\hline \multicolumn{2}{|c|}{ Characteristics } & \multicolumn{3}{|c|}{ Malaria positivity } \\
\hline & & $\begin{array}{l}\text { Microscopy }(\mathrm{n}= \\
51) \%\end{array}$ & $\begin{array}{l}\operatorname{RDT}(\mathrm{n}= \\
61) \%\end{array}$ & $\begin{array}{l}\text { Multiplex real time PCR }(\mathrm{n}= \\
88) \%\end{array}$ \\
\hline \multirow[t]{2}{*}{ Sex } & $\begin{array}{l}\text { Female }(\mathrm{n}= \\
123)\end{array}$ & $15(29.4)$ & 17(27.9) & $35(40)$ \\
\hline & Male $(n=148)$ & $36(70.6)$ & $44(72.1)$ & $53(60)$ \\
\hline \multirow{6}{*}{$\begin{array}{l}\text { Age } \\
\text { group }\end{array}$} & $0-5$ & $5(9.8)$ & $5(8.2)$ & $7(8)$ \\
\hline & $6-15$ & $11(21.5)$ & 13(21.3) & $17(19)$ \\
\hline & $16-25$ & $23(45)$ & $29(47.5)$ & $38(43)$ \\
\hline & $26-40$ & 10(19.6) & 11(18) & $20(23)$ \\
\hline & $41-64$ & $2(3.9)$ & $3(5)$ & $6(7)$ \\
\hline & $\geq 65$ & 0 & 0 & 0 \\
\hline \multirow[t]{2}{*}{ Fever } & Yes $(n=266)$ & 50 & 60 & 87 \\
\hline & No $(n=5)$ & 1 & 1 & 1 \\
\hline \multirow[t]{2}{*}{ Chills } & Yes $(n=253)$ & 50 & 60 & 85 \\
\hline & No $(n=18)$ & 1 & 1 & 1 \\
\hline \multirow[t]{2}{*}{ Headache } & Yes $(n=253)$ & 48 & 58 & 81 \\
\hline & No $(n=18)$ & 3 & 3 & 7 \\
\hline
\end{tabular}

\section{Diagnostic performance of multiplex real time PCR, microscopy and RDT in detecting malaria parasite}

Using microscopy as a reference test, multiplex real time PCR had shown an overall sensitivity, specificity, positive and negative predictive values of $100 \%(95 \% \mathrm{Cl}$ : $93-100), 83.2 \%(95 \% \mathrm{Cl}: 77.57-87.87), 57.95 \%$ (95\% Cl: 46.95-68.4) and 100\% (95\% Cl: 98-100), respectively. Against microscopy, RDT had sensitivity, specificity, PPV and NPV of 98.9\% (95\%Cl: 96.1-99.87), 95\% (95\%Cl: 91.23-97.5), 81.97\% (95\%Cl: 7090.6) and $99.52 \%(95 \% \mathrm{Cl}: 97.38-99.9)$, respectively.

Using multiplex real time PCR as the reference, microscopy had an overall sensitivity, specificity, PPV and NPV of 58\% (95\% Cl: $46.95-68.4), 100 \%$ (95\% Cl: $98-100), 100 \%$ (95\% Cl: $93-100)$ and $83.2 \%$ (95\%Cl: 77.57-87.87), respectively. Against multiplex RTPCR, RDT had sensitivity, specificity, PPV and NPV of $67 \%$ (95\%Cl: 56.2-76.7), 98.9\% (95\%Cl: 96.1-98.87), 96.7\% (95\% Cl: 88.65-99.6) and $86.2 \%(95 \% \mathrm{Cl}:$ 80.77-90.55), respectively (Table 7). 
When microscopy used as a reference test for the identification of $P$. falciparum, multiplex real time PCR shown sensitivity and specificity of $100 \%$ and $83.61 \%$; and $99.2 \%$ and $98.8 \%$ for P.vivax. RDT showed sensitivity and specificity of $100 \%$ and $95.38 \%$ for $P$. falciparum; and $94.44 \%$ and $58.58 \%$ for $P$. vivax, respectively, referring to microscopy (Table 4).

Table 4

The overall performance of microscopy, RDT and multiplex real time PCR for malaria diagnosis at selected sentinel sites, from Dec to March, 2020, Ethiopia.

\begin{tabular}{|c|c|c|c|c|c|}
\hline Characteristics & & $\begin{array}{l}\text { Sensitivity } \\
\text { (Cl: 95\%) }\end{array}$ & $\begin{array}{l}\text { Specificity } \\
\text { (Cl: 95\%) }\end{array}$ & $\begin{array}{l}\text { PPV } \\
\text { (Cl: 95\%) }\end{array}$ & $\begin{array}{l}\text { NPV } \\
\text { (Cl: 95\%) }\end{array}$ \\
\hline \multirow[t]{3}{*}{$\begin{array}{l}\text { Multiplex real time PCR vs } \\
\text { microscopy }\end{array}$} & Overall & $\begin{array}{l}100(93- \\
100)\end{array}$ & $\begin{array}{l}83.2 \\
(77.6- \\
87.9)\end{array}$ & $\begin{array}{l}57.9(47- \\
68.4)\end{array}$ & $\begin{array}{l}100(98- \\
100)\end{array}$ \\
\hline & $\begin{array}{l}P \\
\text { falciparum }\end{array}$ & $\begin{array}{l}100(89- \\
100)\end{array}$ & $\begin{array}{l}83.6(78- \\
88)\end{array}$ & $\begin{array}{l}45.83(34- \\
58)\end{array}$ & $\begin{array}{l}100(98.2- \\
100)\end{array}$ \\
\hline & P. vivax & $\begin{array}{l}94(72.7- \\
100)\end{array}$ & $\begin{array}{l}99.2 \\
(97.2- \\
99.9)\end{array}$ & $\begin{array}{l}90(66.9- \\
98.7)\end{array}$ & $\begin{array}{l}99.6 \\
(97.8-100)\end{array}$ \\
\hline \multirow[t]{3}{*}{ RDT vs microscopy } & Overall & $\begin{array}{l}98(89.6- \\
99.9)\end{array}$ & $\begin{array}{l}95(91- \\
97.5)\end{array}$ & $\begin{array}{l}82(70- \\
0.6)\end{array}$ & $\begin{array}{l}99.5(97- \\
100)\end{array}$ \\
\hline & $\begin{array}{l}P \\
\text { falciparum }\end{array}$ & $\begin{array}{l}100(89- \\
100)\end{array}$ & $\begin{array}{l}95.4 \\
(91.9-7.7)\end{array}$ & $\begin{array}{l}75(59.7- \\
86.8)\end{array}$ & $\begin{array}{l}100(98.4- \\
100)\end{array}$ \\
\hline & P. vivax & $\begin{array}{l}83(58.6- \\
96.4)\end{array}$ & $\begin{array}{l}98.8 \\
(96.6- \\
99.8)\end{array}$ & $\begin{array}{l}83(58.6- \\
96)\end{array}$ & $\begin{array}{l}98.8 \\
(96.6- \\
99.8)\end{array}$ \\
\hline \multirow[t]{3}{*}{$\begin{array}{l}\text { Microscopy vs multiplex } \\
\text { real time PCR }\end{array}$} & Overall & $\begin{array}{l}58(46.9- \\
68.4)\end{array}$ & $\begin{array}{l}100(98- \\
100)\end{array}$ & $\begin{array}{l}100(93- \\
100)\end{array}$ & $\begin{array}{l}83.2 \\
(77.6- \\
87.9)\end{array}$ \\
\hline & $\begin{array}{l}P \\
\text { falciparum }\end{array}$ & $\begin{array}{l}45.8(34- \\
58)\end{array}$ & $\begin{array}{l}100(98.2- \\
100)\end{array}$ & $\begin{array}{l}100(89- \\
100)\end{array}$ & $\begin{array}{l}83.6 \\
(78.3- \\
88.1)\end{array}$ \\
\hline & P. vivax & $\begin{array}{l}89.5 \\
(66.9- \\
98.7)\end{array}$ & $\begin{array}{l}99.6(97.8- \\
9.99)\end{array}$ & $\begin{array}{l}94.4(72.7- \\
9.9)\end{array}$ & $\begin{array}{l}99.2 \\
(97.2- \\
99.9)\end{array}$ \\
\hline \multirow[t]{3}{*}{$\begin{array}{l}\text { RDT vs multiplex real time } \\
\text { PCR }\end{array}$} & Overall & $\begin{array}{l}67(56.21- \\
76.7)\end{array}$ & $\begin{array}{l}98.9(96- \\
99.9)\end{array}$ & $\begin{array}{l}96.7(88.7- \\
9.6)\end{array}$ & $\begin{array}{l}86.2 \\
(80.8- \\
90.6)\end{array}$ \\
\hline & $\begin{array}{l}P \\
\text { falciparum }\end{array}$ & $\begin{array}{l}56.9 \\
(44.7- \\
68.6)\end{array}$ & $\begin{array}{l}98.5 \\
(95.7-9.7)\end{array}$ & $\begin{array}{l}93.2 \\
(81.3-8.6)\end{array}$ & $\begin{array}{l}86.3 \\
(81.2- \\
90.5)\end{array}$ \\
\hline & P. vivax & $\begin{array}{l}84.2 \\
(60.4- \\
96.6)\end{array}$ & $\begin{array}{l}99.2 \\
(97.2- \\
99.9)\end{array}$ & $\begin{array}{l}88.9 \\
(65.3-8.6)\end{array}$ & $\begin{array}{l}98.8 \\
(96.6- \\
99.8)\end{array}$ \\
\hline
\end{tabular}




\section{Result agreement between Microscopy, RDT and multiplex real time PCR}

Overall, species wise multiplex real time PCR identified 69 samples as $P$. falciparum, 16 as $P$. vivax and 3 samples as mixed infection, giving a total of 88 positive samples. From 69 P. falciparum samples, microscopy identified 33 samples as $P$. falciparum, 1 as $P$. vivax and 35 as negative whereas RDT identified 40 samples as $P$. falciparum, 2 as $P$. vivax and the remaining 27 samples as negative. From 16 samples identified as $P$. vivax by multiplex RT PCR, microscopy detected 14 samples as $P$. vivax and 2 samples as negative. RDT identified 14 of them as $P$. vivax, 1 as $P$. falciparum and 1 sample as negative. Three samples were identified as mixed infection (due to $P$. falciparum and $P$. vivax) by multiplex real time PCR whereas only one sample identified as mixed infection by RDT that the remaining two samples were identified as $P$. vivax. None of the samples were detected as mixed infections by microscopy.

Multiplex RT PCR detected Plasmodium infection in all samples that were tested positive by microscopy (51 samples) and additionally 37 samples that were negative by microscopy and 59 from 61 RDT positive samples and 29 from RDT negative samples. Substantial level of \% agreement (86.35) was reported between microscopy and multiplex real time PCR with kappa value of 0.65 . There was observed an agreement of 88.56 (kappa: $0.72 ; P=0.0000$ ) between RDT and multiplex real time PCR. Almost perfect agreement was reported between microscopy and RDT results (kappa value $=0.84, P=0.0000$ ).

At species level, there was little difference in percent agreement between the three techniques in detecting $P$. vivax (multiplex real time-PCR vs RDT $=98.15 \%$ with kappa value of 0.86 , multiplex real time-PCR $v s$ microscopy $=98.89 \%$, kappa value $=0.9$, and microscopy $v s \mathrm{RDT}=97.79 \%$, kappa value $=0.8$ ). However, moderate agreement was reported between microscopy and multiplex real time-PCR $(\%$ of agreement $=$ 85.6 , kappa value $=0.55$ ); substantial agreement between RDT and multiplex real time-PCR (\% of agreement $=87.45$, kappa value $=0.63)$; and almost perfect agreement between microscopy and RDT $(\%$ of agreement $=95.94$, kappa value $=0.8)$ in detecting $P$. falciparum $($ Table 5) . 
Table 5

Percentage of agreements in identification of plasmodium spp. between microscopy, RDT and multiplex real time PCR

\begin{tabular}{|c|c|c|c|c|c|c|c|c|}
\hline \multirow[t]{2}{*}{ Microscopy } & \multicolumn{5}{|c|}{ Multiplex real time PCR } & \multirow{2}{*}{$\begin{array}{l}\text { Percentage } \\
\text { of } \\
\text { Agreement }\end{array}$} & \multirow{2}{*}{$\begin{array}{l}\text { Kappa } \\
\text { value }\end{array}$} & \multirow{2}{*}{$\begin{array}{l}\mathrm{P} \text { - } \\
\text { value }\end{array}$} \\
\hline & $\begin{array}{l}P \\
\text { falciparum }\end{array}$ & $\begin{array}{l}P \\
\text { vivax }\end{array}$ & Mixed & Negative & Total & & & \\
\hline $\begin{array}{l}P_{\text {falciparum }} \\
\text { Pal }\end{array}$ & 33 & 0 & 0 & 0 & 33 & \multirow[t]{5}{*}{$86.35 \%$} & \multirow[t]{5}{*}{0.65} & \multirow[t]{5}{*}{0.0000} \\
\hline P. vivax & 1 & 14 & 3 & 0 & 18 & & & \\
\hline Mixed & 0 & 0 & 0 & 0 & 0 & & & \\
\hline Negative & 35 & 2 & 0 & 183 & 220 & & & \\
\hline Total & 69 & 16 & 3 & 183 & 271 & & & \\
\hline \multicolumn{6}{|l|}{ RDTs result } & \multirow[t]{6}{*}{$88.56 \%$} & \multirow[t]{6}{*}{0.72} & \multirow[t]{6}{*}{0.0000} \\
\hline $\begin{array}{l}P \\
\text { falciparum }\end{array}$ & 40 & 1 & 0 & 2 & 43 & & & \\
\hline P. vivax & 2 & 14 & 1 & 0 & 17 & & & \\
\hline Mixed & 0 & 0 & 1 & 0 & 1 & & & \\
\hline Negative & 27 & 1 & 1 & 181 & 210 & & & \\
\hline Total & 69 & 16 & 3 & 183 & 271 & & & \\
\hline RDT result & \multicolumn{5}{|l|}{ Microscopy } & & & \\
\hline $\begin{array}{l}P \\
\text { falciparum }\end{array}$ & 33 & 2 & 0 & 8 & 43 & \multirow[t]{5}{*}{$94.46 \%$} & \multirow[t]{5}{*}{0.84} & \multirow[t]{5}{*}{0.0000} \\
\hline P. vivax & 0 & 14 & 0 & 3 & 17 & & & \\
\hline Mixed & 0 & 1 & 0 & 0 & 1 & & & \\
\hline Negative & 0 & 1 & 0 & 209 & 210 & & & \\
\hline Total & 33 & 18 & 0 & 220 & 271 & & & \\
\hline
\end{tabular}

\section{Discussion}

Accurate detection and early treatment of every single infection is vital to malaria elimination.

Microscopy and rapid diagnostic tests have been a widely applicable malaria diagnostic tool and helping to achieve malaria control goals. However, malaria elimination requires more sensitive infection detection strategies to halt transmission (17). In this study multiplex real time PCR found to had an excellent sensitivity of $100 \%$ (95\% Cl: $93-100)$ and a good specificity of $83.2 \%$ (95\% Cl: $77.57-87.87)$ compared to microscopy as reference test. In a study done in Toronto the sensitivity of multiplex real time PCR was 
compared to microscopy and they reported $99.4 \%$ of sensitivity which is in line with our result. In this study, the authors showed that multiplex real time PCR is the most analytically sensitive malaria diagnostic tool (18). However, the specificity of this assay has a smaller value than microscopy $(100 \%)$ and RDT (98.5\%). This may be interpreted as microscopy and RDT have more false negative results compared to multiplex real time PCR test. This in turn have an implication on transmission interruption, the ultimate goal of malaria elimination program.

In this study, the positivity rate among malaria suspected febrile patients by multiplex real time PCR was $32.5 \%$ (88/271) which was significantly different from microscopy (19\%) and RDT (22.5\%). Multiplex real time PCR detects all microscopy and $97 \%$ of RDT positive plasmodium infection. The positivity rate by all three diagnostic methods increased among younger age groups and decreased and become zero in older age group, these findings, may be associated with the fact that increased prevalence of asymptomatic infection among these group as a result of developed immunity after repeated exposure. Similarly, a study done in West Arsi Zone, Ethiopia indicated that the overall malaria positivity rate by molecular test was significantly higher than microscopy and RDT tests and the positivity rate among younger age group was highest when determined by microscopy, RDT and molecular test (19). Microscopy and RDT tests under estimate parasite prevalence therefore multiplex real time PCR will be an ideal malaria diagnostic tool that can be used in malaria elimination program since the program demand to observe the true change in parasite prevalence for monitoring it in accordance.

Conventional molecular tests uses multi stage procedures to detect a single parasite species at a time which is labor intensive, time consuming and prone to contamination. In spite of these limitations these molecular testes have been serving as malaria diagnostic tool for research works and evaluation of recently developing diagnostic tools. However, multiplex real time PCR simultaneously detects multiple plasmodium species in a single closed run. In this study, all microscopy identified $P$. falciparum samples were tested positive for $P$. falciparum by multiplex real time PCR. From $18 P$. vivax positive samples by microscopy, multiplex real time PCR identified 14 of them as $P$. vivax and 3 as mixed infections. Microscopy misidentified one $P$. falciparum sample as $P$. vivax which was tested positive for $p$. falciparum by multiplex real time PCR. This discordant result might be explained by the fact that the microscopy test quality mainly influenced by the staining quality, microscopist skill and parasitaemia. A study that conducted in southern Ethiopia was among several studies that revealed microscopy test had lower sensitivity for species identification compared to molecular tests which cause mistreatment of the patients and lead to severe malaria, in this study 14 cases that were microscopically diagnosed as $P$. vivax were found positive for $P$. falciparum when retested by nested PCR (20).

PCR has been considered as a molecular tool for plasmodium detection and species identification. In addition to the detection of low parasitemia and speciation, studies have shown that this technique is robust in identifying mixed species infection that are often undetected and under reported by RDT and microscopy assays. Detecting of mixed infections provides accurate information for patient's treatment and epidemiological studies regarding malaria transmission. (21-23). In the present study, among three mixed infections identified by multiplex real time PCR, RDT detected only one and microscopy none. RDT 
identified as $P$. vivax the rest two and microscopy all three mixed samples as $P$. vivax. This may be due to very low level of parasitaemia of the co-infecting species during mixed infections. Likewise, in a study done in Israel, from ten mixed infections identified by real-time PCR, only one was identified by microscopy and RDT testing(24). In another study conducted in Switzerland to evaluate microscopy and multiplex real time PCR correlation, multiplex qPCR assay correctly identified the species and mixed infections with low levels of parasitaemia but in this study $71 \%$ of mixed infections were misdiagnosed by microscopy (25). In a study done to determine the prevalence of mixed infection using real time PCR in northern Ethiopia from 168 samples, the prevalence of mixed infections were $1.8 \%$ by microscopy and $12.5 \%$ by real time PCR (21). As proved by results from these studies, multiplex real time PCR has the most notable advantages of higher sensitivity to detect mixed infections and to identify species of malaria parasites accurately. Therefore, it is the most convenient malaria diagnostic methods for countries like Ethiopia, where $P$. falciparum and $P$. vivax are co-endemic species, unlike most African countries where $P$. vivax has low or nil endemicity (5).

The RDT test and microscopy had shown lower performance compared to multiplex real time PCR in our study. However, results from both assay shown almost perfect agreement. One sample was tested negative by RDT and it was positive for $P$. vivaxby both PCR and microscopy. This false negative result might be associated with limitations of pLDH-based tests, and these tests had decreased sensitivity at low parasitaemia and performance of detection highly affected by the storage and transportation conditions than HRP2 based tests (26). Another explanation might be the prozone effect of hyper parasitaemia which lead to false negative result in RDT testing (10). In a study of comparative analysis between molecular and serological diagnosis of malaria on large scale in Brazil indicated that the sensitivity and specificity of RDT test compared to multiplex qPCR were found to be $69.56 \%$ and $100 \%$, respectively, which was concordance with our study result (sensitivity of $67 \%$ and specificity of $98.9 \%$ ) (27). There was another study which evaluates the performance of qPCR and RDTs for the diagnosis of malaria in returning traveller from endemic area and qPCR found to have similar sensitivity and specificity with the current study, $99.41 \%$ and $100 \%$, respectively compared to microscopy as reference (18).

Though, identifying malaria into species level have crucial impact on patient managements and transmission interruption, RDT testing are incapable to differentiate and have decreased performance in detecting non-falciparum malaria. In our study the sensitivity of RDT for detecting $P$. vivax were $83.33 \%$ and for $P$. falciparum $100 \%$ compared to microscopy as reference test. This result was concordance with the performance of similar RDT format in a study done by Felekeet al. but lower sensitivity was documented in study conducted by Moges et al.(28, 29).

In this study, nearly all (87/88) multiplex real time PCR positive cases were from patients presented with self-reported fever which was large proportion when compared to febrile cases identified by RDT and microscopy. The negative predictive value of multiplex real time PCR found to be $100 \%$ using microscopy as a reference test. Diagnostic tool with high negative predictive value reduce the probability of missing infection in individuals, means when the patient is malaria negative using multiplex real time PCR, 
plasmodium infection could be ruled out with high certainty. This quality of PCR makes it an ideal diagnostic tool to be used in malaria elimination endeavors than RDT and microscopy which were found to have high positive predictive value and low negative predictive value in current study. RDT and microscopy malaria diagnosing increase the probability of missing plasmodium infection which have negative impact on transmission interruption since all infected individuals considered as potentially infectious and thus potentially able to contribute to ongoing transmission(1) .

Multiplex real time PCR identified 69 malaria cases as $P$. falciparum infection, 16 as vivax and 3 as mixed infections from the total of 271 symptomatic malaria suspected patients in this study. Of these malaria cases large number of $P$. falciparum cases missed by both RDT and microscopy testing however, all three methods showed perfect agreement in $P$. vivax species identification. Additionally, the specificity of multiplex real time PCR for $P$. vivax identification (98.8\%) were much better than $P$. falciparum identification (83.61\%) when compared to microscopy as reference test. This is probably due to the presence of all erythrocytic stage of $P$. vivax in peripheral bloods that increase parasitic densities in the blood of symptomatic patients but $P$. falciparum causes cytoadherence and sequestration of infected erythrocytes that reduced parasite densities from peripheral blood (30). Using microscopy as reference test, multiplex real time PCR showed excellent sensitivity for both P. falciparum (100\%) and P. vivax $(99.2 \%)$ species identification in this study. Which is closely related to the finding of study done at Bangladesh on clinically suspected malaria patients that the sensitivity of real time PCR for $P$. falciparum and $P$. vivax identification using microscopy as a gold standard were $97.1 \%$ and $95.2 \%$, respectively (31).

Substantial numbers of $P$. falciparum infection was detected by multiplex real time PCR than microscopy and RDT methods. This might be explained as follows, in this study varATS TaqMan PCR method were used for $P$. falciparum detection which is a highly sensitive PCR primers for malaria than 18S rRNA based PCR which were also used in a current study for the detection of $P$. vivax (34). Another reason may be due to the biology of the parasite in which the parasite has a tendency to sequestrated in the organs during its life cycle and unable to detect by RDTs and microscopy.

\section{Conclusions}

Multiplex real time PCR was the most sensitive malaria diagnostic method that can be used in malaria elimination program. It had an advanced performance in species identification and mixed infection detection than microscopy and RDT in low malaria transmission settings and showed better performance in detection of Plasmodium infection among febrile patients. Therefore, this assay can be used for epidemiological and community based prevalence studies and for verification of elimination in areas where malaria elimination was launched. However, microscopy and RDT still have an acceptable performance to be used as a malaria diagnostic tool in health facilities for patient's treatment due to the fact that they are affordable and easily performed diagnostic methods.

\section{Abbreviations}


DBS Dried Blood Spot

EDTA Ethylene Diamine Tetra Acetic Acid

EPHI Ethiopian Public Health Institute

FMOH Federal Ministry of Health

MIS Malaria Indicator Survey

NMSP National Malaria Strategic Plan

NPV Negative predictive value

PCR Polymerase Chain Reaction

PPV Positive predictive value

RDT Rapid Diagnostic Test

WHO World Health Organisation

\section{Declarations}

\section{Acknowledgements}

We would like to thank Ethiopian Public Health institute for financial support. We would also like to thank the study participants and data collectors.

\section{Authors' contributions}

$M B, A d A, D N$ and $M W$ contributed in conceptualization, design, and protocol development. MB, AdA and $L N$ analyzed data. MB and AdA optimized, performed laboratory tests and drafted the manuscript. MB, MW, DN, BG, LN, GT, AA, AW and AdA read and commented on the manuscript. All authors read and approved the final manuscript.

\section{Funding}

Funding for field data collection and training was provided by the Ethiopian Federal Ministry of Health.

\section{Availability of data and materials}

The dataset and materials used for the study are kept in a safe place in EPHI data management center.

\section{Ethical approval and consent to participants}


The study protocol was reviewed and approved by the Ethiopian Public Health Institute -IRB Office, Addis Ababa, Ethiopia.

\section{Consent for publication}

All authors have read and agreed to publish this article.

\section{Competing interests}

The authors declare that they have no competing interests.

\section{Author details}

${ }^{1}$ Department of Medical Laboratory Sciences, College of Health Sciences, Addis Ababa University.

${ }^{2}$ Malaria and Neglected Tropical Diseases Research Team, Ethiopian Public Health Institute, P.O. Box: 1242, Addis Ababa, Ethiopia.

\section{References}

1. WHO. World malaria report 2020. Geneve, Swizerland.

2. Cowman AF, Healer J, Marapana D, Marsh K. Malaria: Biology and Disease. Cell [Internet]. 2016;167(3):610-24. Available from: http://dx.doi.org/10.1016/j.cell.2016.07.055.

3. WHO Report. WHO I The World malaria report 2018 [Internet]. WHO. 2018. Available from: https://www.who.int/malaria/publications/world-malaria-report-2018/en/.

4. Animut A, Lindtjørn B. Use of epidemiological and entomological tools in the control and elimination of malaria in Ethiopia. Malar J [Internet]. 2018;17(1):1-8. Available from: https://doi.org/10.1186/s12936-018-2172-1.

5. Taffese HS, Hemming-Schroeder E, Koepfli C, Tesfaye G, Lee MC, Kazura J, et al. Malaria epidemiology and interventions in Ethiopia from 2001 to 2016. Infect Dis Poverty. 2018;7(1):1-9.

6. FMOH. National Strategic Plan for Malaria Prevention Control and Elimination in Ethiopia. 20112015.2010;2015 (August 2010):76. Available from: http://www. national planning cycles.org/sites/default/files/ country docs /Ethiopia/ ethiopia malaria national strategic plan 2011-2015 130810. pdf.

7. President's Malaria Initiative Ethiopia. Malar Oper Plan FY 2018. 2018;2-9.

8. $\mathrm{MOH}$. Ethiopia malaria elimination strategic plan: 2021-2025. 2021;2021-5.

9. Adugna A. Malaria inDemography and Health.Org [Internet]. 2006;Lesson 14. Available from: www.EthioDemographyAndHealth.

10. Tedla M. A focus on improving molecular diagnostic approaches to malaria control and elimination in low transmission settings: Review. Parasite Epidemiol Control [Internet]. 2019;6:e00107. Available from: https://doi.org/10.1016/j.parepi.2019.e00107. 
11. Zheng Z, Cheng Z. Advances in Molecular Diagnosis of Malaria. Adv Clin Chem. 2017;80:155-92.

12. FMOH. National Malaria Guidelines. Natl Malar Giuideline. 2018;4th edition:155.

13. Mitsakakis K, Hin S, Müller P, Wipf N, Thomsen E, Coleman M, et al. Converging human and malaria vector diagnostics with data management towards an integrated holistic one health approach. Int $\mathrm{J}$ Environ Res Public Health. 2018;15(2).

14. Santana-Morales MA, Afonso-Lehmann RN, Quispe MA, Reyes F, Berzosa P, Benito A, et al. Microscopy and molecular biology for the diagnosis and evaluation of malaria in a hospital in a rural area of Ethiopia. Malar J. 2012;11:1-7.

15. Zaidi M, Hospital LN, Waseem H, Fahim M, Ansari A, Hospital LN, et al. SAMPLE SIZE ESTIMATION OF DIAGNOSTIC TEST STUDIES IN. Proc 14th Int Conf Stat Sci. 2016;29(February 2018):239-46.

16. Haanshuus CG, Mørch K, Blomberg B, Strøm GEA, Langeland N, Hanevik K, et al. Assessment of malaria real-time PCR methods and application with focus on low-level parasitaemia. PLoS One. 2019;14(7):e0218982.

17. Zimmerman PA, Howes RE. Malaria diagnosis for malaria elimination. Curr Opin Infect Dis. 2015;28(5):446-54.

18. Khairnar K, Martin D, Lau R, Ralevski F, Pillai DR. Multiplex real-time quantitative PCR, microscopy and rapid diagnostic immuno-chromatographic tests for the detection of Plasmodium spp: Performance, limit of detection analysis and quality assurance. Malar J. 2009;8(1):1-17.

19. Golassa L, Enweji N, Erko B, Aseffa A, Swedberg G. Detection of a substantial number of submicroscopic Plasmodium falciparum infections by polymerase chain reaction: A potential threat to malaria control and diagnosis in Ethiopia. Malar J. 2013;12(1):1-10.

20. Mekonnen SK, Aseffa A, Medhin G, Berhe N, Velavan TP. Re-evaluation of microscopy confirmed Plasmodium falciparum and Plasmodium vivax malaria by nested PCR detection in southern Ethiopia. Malar J [Internet]. 2014;13(1):1-8.

21. Tajebe A, Magoma G, Aemero M, Kimani F. Detection of mixed infection level of Plasmodium falciparum and Plasmodium vivax by SYBR Green I-based real-Time PCR in North Gondar, north-west Ethiopia. Malar J. 2014;13(1):1-8.

22. Ehtesham R, Fazaeli A, Raeisi A, Keshavarz H, Heidari A. Detection of mixed-species infections of Plasmodium falciparum and Plasmodium vivax by Nested PCR and rapid diagnostic tests in Southeastern Iran. Am J Trop Med Hyg. 2015;93(1):181-5.

23. Krishna S, Bharti PK, Chandel HS, Ahmad A, Kumar R, Singh PP, et al. Detection of mixed infections with plasmodium spp. by PCR, India, 2014. Emerg Infect Dis. 2015;21(10):1853-7.

24. Grossman T, Schwartz E, Vainer J, Agmon V, Glazer Y, Goldmann D, et al. Contribution of real-time PCR to Plasmodium species identification and to clinical decisions: a nationwide study in a nonendemic setting. Eur J Clin Microbiol Infect Dis [Internet]. 2017;36(4):671-5. Available from: http://dx.doi.org/10.1007/s10096-016-2844-0.

25. Dormond L, Jaton-Ogay K, De Vallière S, Genton B, Bille J, Greub G. Multiplex real-time PCR for the diagnosis of malaria: Correlation with microscopy. Clin Microbiol Infect. 2011;17(3):469-75. 
26. Diallo MA, Diongue K, Ndiaye M, Gaye A, Deme A, Badiane AS, et al. Evaluation of CareStart ${ }^{\text {TM }}$ Malaria HRP2/pLDH (Pf/pan) Combo Test in a malaria low transmission region of Senegal. Malar J. 2017;16(1):328.

27. Lima GFMC, Levi JE, Geraldi MP, Sanchez MCA, Segurado AA, Hristov AD, et al. Malaria diagnosis from pooled blood samples: Comparative analysis of real-time PCR, nested PCR and immunoassay as a platform for the molecular and serological diagnosis of malaria on a large-scale. Mem Inst Oswaldo Cruz. 2011;106(6):691-700.

28. Feleke DG, Tarko S, Hadush H. Performance comparison of CareStart ${ }^{\mathrm{TM}}$ HRP2/pLDH combo rapid malaria test with light microscopy in north-western Tigray, Ethiopia: A cross-sectional study. BMC Infect Dis. 2017;17(1):1-7.

29. Moges B, Amare B, Belyhun Y, Tekeste Z, Gizachew M, Workineh M, et al. Comparison of CareStart HRP2/pLDH COMBO rapid malaria test with light microscopy in north-west Ethiopia. Malar J. 2012;11:1-6.

30. Adams JH, Mueller I. The Biology of Plasmodium vivax. 2017;1-12.

31. Alam MS, Mohon AN, Mustafa S, Khan WA, Islam N, Karim MJ, et al. Real-time PCR assay and rapid diagnostic tests for the diagnosis of clinically suspected malaria patients in Bangladesh. Malar $\mathrm{J}$. 2011;10:1-9. 